${ }_{20}$ Burnett, D. S., Fowler, W. A, and Hoyle, F., Geochim. Cosmochim. Acta, $29,1209(1965)$.

${ }^{21}$ Richter, J., and Tonner, K. F., Z. Astrophys., 6\%, 155 (1967).

${ }^{22}$ Lambert, D. L., and Mallia, E. A., Astrophys. Lett., 1, 85 (1968).

${ }^{23}$ Peach, J. W., Mon. Not. Roy. Astron. Soc., 139, 403 (1968).

${ }^{24}$ Grevesse, N., Solar Phys., 5, 159 (1968).

is Stellmacher, G., and Wichr, E., Mitt. Astron. Ges. (Hamburg), 25, 198 (1968).

${ }^{26}$ Strock, L. W., Nachr. Ges. Wiss. Göttingen, Fachgr. IV, 1, 171 (1936).

${ }^{27}$ Bonsack, W. K., Astrophys. J., 133, 340 (1960).

${ }^{28}$ Wallerstein, G., Herbig, G. H., and Conti, P. S., Astrophys. J., 141, 610 (1965).

2.' Danziger, I. J., Astrophys, J, 150, 733 (1967)

${ }^{30}$ Krankowsky, D., and Müller, O., Geochim. Cosmochim. Acta, 31, 1833 (1967).

\section{Consequences of Very Small Planetary Magnetic Moments}

Palakomagnetic samples show a number of polarity transitions in the Earth's dipole field, and there seem to have been marked evolutionary changes in flora and fauna near the time of several of the reversals ${ }^{1}$. Suggestions that when the magnetic dipole moment decayed during a reversal, there would be an increase in radiation producing a sudden increase in the rate of evolution ${ }^{2}$ seem to have been discarded without considering whether the atmospheric mass would be reduced ${ }^{1,3}$. Mariner V observations show that Venus now has a dipole moment of zero or very near to zero ${ }^{4}$, so that the atmosphere of Venus may show features which can be explained by erosion and modification by the solar wind. For example, Jastrow ${ }^{5}$ has stated that the expected dissociation of $\mathrm{CO}_{2}$ and $\mathrm{H}_{2} \mathrm{O}$ in the atmosphere of Venus should have produced high altitude layers of electrons and atomic oxygen which were not found.

Consideration of the erosion of a planetary atmosphere by the solar wind when the dipole moment is near zero suggests that little or no $\mathrm{O}^{+}$should be observed for Venus or Mars above the ionosphere, that enhancement of the cosmic radiation on the Earth cannot be achieved easily and that the solar radiant energy might increase in certain regions of the Earth's atmosphere.

Interaction of the solar wind with a planet's magnetosphere results in a boundary called the magnetopause, inside which the atmosphere is protected from erosion by the solar wind. Outside the bow shock on the sunward side of the magnetopause, the solar wind will rapidly sweep away all ions formed. Tons formed in the disturbed region between the inner edge of the magnetopause and the bow shock will also be lost, although the loss rate may be slower than outside the bow shock. Inside the magnetopause there will be no solar wind erosion. As the strength of a planet's magnetic field decreases, the magnetopause moves towards the planet's surface. At present the magnetopause for the Earth is at about ten Earth radii in the subsolar direction, well outside the Earth's atmosphere. The maximum value of the dipole moment of Venus measured so far is too small to keep the solar wind off the planet's surface; however, the Mariner V observations ${ }^{5}$ show that there is a daytime ionosphere at $130 \mathrm{~km}$ altitude with a thickness at half intensity of $20 \mathrm{~km}$. The interplanetary field cannot penetrate a sufficiently conducting ionosphere and is compressed on top of the ionosphere to form a magnetopause. The pressure on the ionosphere is transferred to the lower neutral atmosphere.

During a reversal of the Earth's magnetic field, the solar wind should compress the Earth's ionosphere to the same atmospheric pressure as for Venus. 'Thus the Earth's ionosphere would be at about $85 \mathrm{~km}$ altitude and the atmosphere above 100 to $120 \mathrm{~km}$ would be above the inner edge of the magnetopause. By the same argument the ionosphere of Mar's should be compressed to about $50 \mathrm{~km}$ altitude. According to the reversal curves of dipole moment shown by $\mathrm{Cox}^{1}$ the dipole moment may be small enough for the solar wind to erode the Earth's atmosphere for about one solar cycle (11 yr). The great variability in solar activity from one solar cycle to another means that effects on the Earth's atmosphere may be drastically different from one reversal to another.

During a reversal, the solar wind should sweep away all ions produced in the Earth's atmosphere above 100 to $120 \mathrm{~km}$ altitude. Using an ion production rate of $5 \times 10^{10}$ ion pairs $\mathrm{cm}^{-2} \mathrm{~s}^{-1}$ and allowing for effects such as recombination, night and obliquity by introducing a reduction by a factor of six, a loss of the order of $10^{-4} \mathrm{~g} \mathrm{~cm}^{-2}$ during the solar cycle is obtained. Because most of the $\mathrm{O}^{+}$is produced at a level above the inner edge of the magnetopause, $\mathrm{O}^{+}$would be effectively eliminated from the Earth's atmosphere. The loss of $10^{-4} \mathrm{~g} \mathrm{~cm}^{-2}$ of ions in a solar cycle would only reduce the amount of atmosphere shielding from cosmic radiation by $10^{-7}$ and thus would not lead to any significant increase in cosmic radiation.

There might be removal of some of the neutral atmosphere, and there are a number of possible mechanisms for coupling the solar wind to the neutral atmosphere. Between 1 and 10 per cent of the atmosphere (10 to $10^{2} \mathrm{~g} \mathrm{~cm}^{-2}$ ) would have to be removed during the few years at reversal significantly to increase cosmic radiation at the surface. Thus the removal rate must be a factor of $10^{5}$ to $10^{6}$ larger than the ion removal, which seems difficult to achieve. If 10 to $10^{2} \mathrm{~g} \mathrm{~cm}^{-2}$ were removed during each reversal, an adequate replenishment mechanism would be required. But the loss of $10^{-4} \mathrm{~g} \mathrm{~cm}^{-2}$ of ions in a solar cycle may alter significantly the penetration and absorption of solar ultraviolet radiation, resulting in a modification of the constituents, chemistry, temperature, albedo, scale height and motion of the atmosphere.

It may be easier to explain the structure of the atmosphere of Venus if the ions produced above 170 to $200 \mathrm{~km}$ altitude are removed by the solar wind. The loss of atomic oxygen below the Venus magnetopause is explained by McElroy ${ }^{6}$. Atomic oxygen above the magnetopause would be photoionized and lost to the solar wind. A similar argument can explain the failure to observe atomic oxygen in the atmosphere of Mars. In the case of Mars, the ionosphere, as interpreted from the Mariner IV data ${ }^{6}$, would have to be readjusted; the structure of the ionosphere seems. however, to be in considerable doubt?

We thank Dr Martin Watt for helpful discussions.

Billy M. McCormac

JoHN E, Evans

Lockheed Palo Alto Research Laboratory,

3251 Hanover Street,

Palo Alto, California 94304.

Received April 28, 1969.

${ }^{1}$ Cox, A., Science, 163, 237 (1969).

${ }^{2}$ Uffen, R. J., Nature, 198, $143(1963)$

${ }^{3}$ Harrison, C. G. A., Nature, 217,46 (1968).

- Snyder, C. W., Science, 158, 1665 (1967).

5 Jastrow, R., Science, 160, 1403 (1968).

${ }^{6}$ MeElroy, M. B., J. Geophys. Res., 74, 29 (1969).

Harrington, J. V., Grossi, M. D., and Langworthy, B. M., J. Geophys. Res., 73, 3039 (1968).

\section{Records obtained from the Trials of a Long Range Side-scan Sonar (GLORIA Project)}

IN 1958 the National Institute of Oceanography developed a narrow beam sideways looking sonar for underwater geology and fish detection' ${ }^{1}$ based on earlier work first reported on by Chesterman, Clynick and Stride ${ }^{2}$. During the past 10 years it has proved to be a valuable tool in studying the materials and relief of the sea floor at continental shelf depths. For some years it has been supplied to research laboratories and geophysical prospector's by instrument manufacturers in Britain and elsewhere. 